\title{
Blogging about Family Building (Infertility, Pregnancy Loss, Adoption, Pregnancy, Trying to Conceive): Content and Blogging Motivations
}

\author{
Sara L. Sohr-Preston ${ }^{1}$, Alyssa Lacour ${ }^{2}$, Tyler Brent ${ }^{3}$, Timothy Dugas ${ }^{1}$, Lauren Jordan ${ }^{1}$ \\ ${ }^{1}$ Department of Psychology, Southeastern Louisiana University, Hammond, LA, USA \\ ${ }^{2}$ Department of Psychology, University of Louisiana at Lafayette, Lafayette, LA, USA \\ ${ }^{3}$ School of Social Work, Southern University at New Orleans, New Orleans, LA, USA \\ Correspondence: Sara L. Sohr-Preston, Department of Psychology, Southeastern Louisiana University, Hammond, LA, \\ USA
}

Received: January 5, 2016 Accepted: January 18, 2016 Online Published: February 26, 2016

doi:10.11114/smc.v4i1.1281 URL: http://dx.doi.org/10.11114/smc.v4i1.1281

\begin{abstract}
To better understand the content and process of family building blogging, two interrelated studies took place. First, blogs with posts during a designated week were analyzed for content. Then, hosts of active blogs were invited to complete a survey on their blog content and reasons for blogging. Results of both content analysis and survey indicated a variety of content appearing in such blogs, with children, pregnancy, attempts to conceive, and negative emotional experiences related to those emerging as popular topics. To identify motivations for blogging about such content, a principal components analysis (PCA) suggested seven different blogging motivations: gaining attention, benefitting others, becoming a career blogger, entertaining others, documenting life events, self-expression, and forming an online social support network. To further explore blogging motivations, subgroups of bloggers (those having experienced infertility, those having experienced pregnancy loss, and those already parenting) were compared on their ratings of the seven blogging motivations with multivariate analysis of variance (MANOVA). While results mostly supported consistency across subgroups in motivations, there was a significant effect for infertility experience on ratings for educating others and self-expression. This investigation represents the first known attempt to capture what information is included in the growing subgenre of family building blogs and what reasons inspire bloggers to create and maintain them.
\end{abstract}

Keywords: blogging, infertility, pregnancy, miscarriage, content analysis

\section{Introduction}

\subsection{What are Family Building Blogs?}

For both healthy and sick individuals, the Internet has expanded opportunities for dissemination of health information and social support for health struggles through a variety of vehicles (Himmel, Meyer, Kochen, \& Michelmann, 2005; Mclellan, 1998), one of them being weblogs, more commonly called blogs (Rains \& Keating, 2011). A blog is a website presenting dated entries, or posts, containing either text, images, or audio/video and typically listed in reverse-chronological order (Huang, Shen, Lin, \& Chang, 2007; Miller \& Pole, 2010; Nardi, Schiano, Gumbrecht, \& Swartz, 2004b; Schiano, Nardi, Gumbrecht, \& Swartz, 2004), most taking the form of an online diary (Nardi, Schiano, \& Gumbrecht, 2004a) with fairly brief posts (Schiano et al., 2004). Initially, keeping a blog required knowledge of programming, but with introduction of user-friendly free services such as Blogger and WordPress, anyone with internet access can start and maintain a blog (Huang et al., 2007; Miller \& Pole, 2010).

Bloggers frequently form niche networks focused on a single topic or array of topics (Knoll \& Bronstein, 2014; Miller \& Pole, 2010; Ratliff, 2009), one of these recognized networks commonly referred to as the infertility blogging network (Knoll \& Bronstein, 2014). Infertility (or "IF") blogging may be currently a misnomer, however, since the label has been applied to blogging about a variety of topics not necessarily discussed within the context of infertility (i.e., pregnancy loss, adoption, pregnancy, and trying to conceive). For this reason, we use the more inclusive term family building $(F B)$ bloggers to describe this community since its members, regardless of fertility status or history, all discuss attempts to start, grow, or maintain families. 


\subsection{Why Blog about Health or Personal Issues?}

Past research has supported the utility of blogging and other online sources of support for health problems to disseminate and obtain information (Gold, Boggs, Mugisha, \& Palladino, 2012; Malik \& Coulson, 2008; Welbourne, Blanchard, \& Boughton, 2009; Ziebland \& Wyke, 2012), reduce perceived isolation and build an online social support network (Gold et al., 2012; Miller \& Pole, 2010; Rains \& Keating, 2011; Ressler, Bradshaw, Gualtieri, \& Chui, 2012). Online support resources like blogs, message boards, and online support groups offer some distinct advantages over face-to-face, in-person, or, to use common internet terminology, "in real life" (IRL) attempts to gain social support or information. Online resources, for example, offer anonymity craved by some experiencing sensitive or not often openly discussed situations (such as early pregnancy, trying to conceive when single or in a same-sex relationship, infertility, pregnancy loss, or adopting), 24-hour availability, and means to connect with or gain information from individuals worldwide without transportation barriers (Malik \& Coulson, 2008; Welbourne et al., 2009). Furthermore, these advantages may increase heterogeneity within an online support network, resulting in a potentially richer array of information and experiences to absorb (Welbourne et al., 2009).

On another note, some may seek out FB blogging out of dissatisfaction with the advice, information, or support encountered IRL. Infertile couples or individuals, for example, typically receive information on the medical and technical aspects of diagnosis and treatment while psychosocial support is largely neglected (Cousineau et al., 2004). In addition, infertility and pregnancy loss may carry a stigma making individuals reluctant to approach friends and family IRL to express their feelings, share life experiences, or seek social support (Slade, O'Neill, Simpson, \& Lashen, 2007). Blogs, as a "pull" instead of "push" technology (i.e, only those wishing to read their accounts must do so, and only when convenient; Nardi et al., 2004b; Schiano et al., 2004), allows those struggling with FB to accomplish these goals in a non-demanding manner. For these reasons, launching and maintaining a FB blog may hold special appeal.

While research on blogs discussing infertility and other FB topics is currently limited, it has been documented that women diagnosed with infertility are more active users of the Internet than women without infertility (Okamura, Bernstein, \& Fidler, 2002; Weissman, Gotlieb, Ward, Greenblatt, \& Casper, 2000). Some past studies have targeted other forms of online support such as online message boards devoted to infertility. Such research has revealed particular advantages of online support resources for infertility, including options for seeking highly specialized information about particular diagnoses, procedures, and medications (Welbourne et al., 2009). Blogs differ from message boards in that readers probably must put forth more effort to find blogs covering topics relevant to their own situations. While message boards are often already categorized by topic and found within a larger informational site, blogs may cover multiple topics and not be linked to another "main" site. Readers often locate a new blog by seeing a link on another blog, profile, or homepage (Nardi et al., 2004b). Some categorized online lists of FB blogs are available, with one extensive list associated with the website, Stirrup Queens (http://www.stirrup-queens.com/a-whole-lot-of-blogging-brought-to-you-sorted-and-filed/), which is sorted into "rooms" by the categories of Adoption Room, Donor Gamete and Surrogacy Room, Living Child-Free Room, Loss Room, General Infertility and Treatments, Diagnostics Room, Situation Room, General Pregnancy or Parenting Room, and Miscellaneous Room (Ford, 2015). Curious readers may explore such aggregator sites and essentially shop for blogs. Furthermore, individual bloggers may include a blogroll (i.e., listing of blog links) in their sidebar, thus potentially introducing readers to blogs covering similar topics. In fact, Miller and Pole (2010) have suggested that the option to include blogrolls may be particularly conducive to forming online social support networks.

Individuals and couples struggling with starting or expanding a family may in these ways discover the FB blogosphere (i.e., a collection of blogs and their readers and connections) and experience benefits from documenting their struggles and achievements and from reading the accounts of bloggers in similar situations. In fact, blogs about pregnancy, birth, and fertility are beginning to be recognized as information resources, while still being described as potentially substandard or contradictory to more evidence-based yet still easily accessible information resources (Alati-it \& Pinches, 2014; Okamura et al., 2002; Wischmann, 2008). Despite precautions against the credibility of information acquired from blogs, blogs may hold special appeal over contact with medical professionals for those seeking to safely connect with others experiencing struggles similar to their own (Neal \& McKenzie, 2011).

\subsection{What Else Can Be Learned?}

The available literature indicates FB blogging is a viable niche network with many potential appealing features, yet research has yet to address more specific aspects of family building blogging. For these reasons, the current investigation targeted blogs devoted partially or fully to the process of family building, including infertility, pregnancy loss, adoption, pregnancy, and trying to conceive. The study proceeded in two parts. First, the investigators conducted a content analysis of one week's posts of active FB blogs. Then, a survey was made available to bloggers who had posted 
within the previous year. Each part was conducted to achieve the general goal of bringing attention to this burgeoning sub-genre of blogging. In addition, this investigation was designed to address the following research questions.

\subsubsection{How Accessible and Active Are FB Blogs?}

An initial step involved documenting how many FB blogs would be both accessible to the curious reader and contain recent content. Since many blogging services allow users to restrict or password-protect their blogs and blogs are easily started and abandoned, we were uncertain as to how often a reader would come across a restricted or inactive blog.

\subsubsection{What Sort of Content Would a Reader Encounter upon Finding an Open and Active FB Blog?}

We sought to provide researchers and health professionals with an idea of what topics those coming across these blogs would encounter. Past descriptions of the FB blogging community have generally been undertaken by popular press writers or journalists (see Miller, 2008) or academics in the humanities (see Striff, 2005). Since, to our knowledge, no attempt has yet been made to describe the specific topics commonly appearing in these blogs, we set out to create such a description.

\subsubsection{Who Tends to Keep a FB Blog?}

We also hoped to increase understanding of FB bloggers themselves. Previous subjective descriptions suggest a community of mostly White and largely affluent women keeping personal journal-style FB blogs (Miller, 2008). We aimed to verify or refute this generalization regarding demographic features of FB bloggers.

\subsubsection{What Motivates an Individual to Blog about Family Building?}

Previous descriptions convey FB bloggers creating a safe space to document their personal struggles involved in FB (Striff, 2005) and sometimes achieving celebrity status (Belkin, 2011) or earning income from blogging (Murphy, 2011). Most blogs have fairly small audiences (Huang et al., 2007; Nardi et al., 2004b), however, making fame or fortune seem unlikely motivations for blogging. According to Nardi and colleagues (2004b), five common and potentially overlapping motivations for blogging are documenting life experiences, providing commentary and opinions, expressing emotions, articulating ideas through writing, and forming and maintaining community forums. Huang and colleagues (2007) expanded this line of work and slightly altered their categorization of blogging motivations such that articulating ideas through writing and expressing emotions were combined into a single category of self-expression, and a category was added for information searching. It remains to be seen whether any of these observed blogging motivations apply to FB bloggers. Because of this gap in the literature, once FB blogs were identified and analyzed for content, we reached out to FB bloggers to explore and derive categories of blogging motivation.

\subsubsection{Do Blogging Motivations Differ among Subgroups of FB Bloggers?}

Finally, since FB bloggers do not necessarily make up a completely homogenous community, we compared blogging motivation across different subgroups of FB bloggers (those having experienced infertility, those having experienced pregnancy loss, and those who were already parents) to explore whether any differences would emerge. Taken together, we hoped addressing these research questions would allow for increased awareness and understanding of the FB blogging community and its potential benefits to both readers and bloggers.

\section{Method}

\subsection{Participants}

Blogs to be coded for FB content were identified using Google searches for keywords generated by research team members, and included "infertility blog," "IF blog," ("IF" is a commonly-used abbreviation for infertility within online communities) "trying to conceive blog," "TTC blog," ("TTC" is a common online abbreviation for trying to conceive) "miscarriage blog," "recurrent pregnancy loss blog," "pregnancy blog," and "adoption blog." New search terms were added as team members were exposed to more blogs. For instance, "recurrent pregnancy loss blog" was added to the list of search terms after team members had begun to visit blogs linked on other sites so as to copy their web address and noticed some bloggers referred to their blogs as such. "TTC blog," "IF blog," "miscarriage blog," and "adoption blog" were added based on common headings viewed within blogrolls. In addition, whenever a Google search directed the researchers to a list of blogs, all blogs on the list were examined as potential blogs to be coded. Searching and removal of duplicates resulted in a list of 3527 blogs.

For blogs labeled "active" (i.e., at least one post in the year preceding coding), invitations for bloggers to complete a survey were sent via e-mail or blog comment (depending on which was possible given blog setup). Some invitations could not be sent because comments had been closed or limited to invited readers or no e-mail address was listed. Out of 1686 bloggers with active blogs, 291 participated in the survey. Relevant to the third research question and consistent with past descriptions of FB bloggers (see Miller, 2008), most bloggers reported being female $(98.88 \%$ ) and the sample was predominately White $(93.85 \%)$. The average blogger age was 35.68 (median $=35$; mode $=30 ; S D=6.09$; range $=$ 
19 - 59). The vast majority (98.13\%) of bloggers reported hosting single-author, personal journal types of blogs. In terms of anonymity, $37.22 \%$ reported identifying themselves in their blogs, while $48.50 \%$ reported trying to stay anonymous but including some details potentially revealing their identities. Only $14.29 \%$ reported having no identifying information whatsoever on their blogs. About one-third (36.23\%) had been blogging more than five years, and $8.30 \%$ had been blogging less than one year.

Many of the bloggers were parents (58.08\%), with $16.49 \%$ of the total sample being adoptive parents, $3.09 \%$ reporting being foster parents, and $3.09 \%$ stepparents. The sample included parents of multiples $(8.25 \%)$, and parents of children with special needs (7.22\%). Some same-sex parents (2.06\%) and single parents (2.75\%) were represented.

The majority of the sample $(62.89 \%)$ had experienced infertility. A smaller number had experienced miscarriage or pregnancy loss $(39.52 \%)$, infant death $(5.15 \%)$, or death of a child $(0.34 \%)$. A few participants reported trying to conceive while single $(1.72 \%)$. Some respondents were medical professionals $(5.50 \%)$. While the numbers were small (all less than 1.00\%), there were surrogate mothers, sperm donors, and egg donors in the sample.

\subsection{Measures}

\subsubsection{Coded Blog Content}

Coders recorded the frequency of posts, comments, and links to other blogs within a one-week period. They additionally marked the presence or absence, using Yes/No items, of mention of the topics listed in Table 1. The coding manual can be obtained from the corresponding author for further information on decision rules and included procedures, drugs, therapies, etc.

Coder training involved at least one 30-minute lecture and demonstration of correct use and interpretation of the coding manual. Based on pilot coding and a research team member's preexisting familiarity with the FB blogging community as a former blogger, coders were introduced to common abbreviations, drug names, procedures, pregnancy terms, and steps in the adoption process likely to appear in blogs to be coded. Five blogs were randomly selected using a random number generator applied to the alphabetized master list of blogs. These five blogs were initially coded by the principal investigator (i.e., master coder) and designated as training assignments. Trainees achieving adequate inter-rater reliability (IRR) with the master coder (based on average Cohen's kappa and intraclass correlation coefficient values of at least .70) were allowed to receive coding assignments. Coders failing to achieve adequate reliability would have been required to complete another set of training assignments, but no coders required this additional practice.

After training, approximately $20 \%$ of blogs were still assigned to two or more coders to assess IRR. Reliability for Yes/No items was computed with Cohen's kappa, and reliability of continuous items (i.e., number of posts, number of comments, and number of links to other blogs) was calculated using intraclass correlation coefficients (ICC) as recommended in Rousson, Gasser, and Seifert (2002). IRR values are summarized in Table 1. Kappa values ranged from .62 to .86 , all falling within the "substantial agreement" or "almost perfect agreement" range (Viera \& Garrett, 2005). Furthermore, ICC values were high, ranging from .81 to 1.00. Therefore, the values in Table 1 support good to excellent IRR for coding.

Table 1. Inter-rater reliability of content coding

\begin{tabular}{ll}
\hline Area of content & kappa \\
\hline Number of posts & ICC \\
Number of comments & .99 \\
Number of links to other blogs & 1.00 \\
Attempts to get pregnant & .81 \\
Attempts to adopt & .79 \\
Being pregnant & .71 \\
Miscarriage or stillbirth & .82 \\
Assisted reproductive technology (ART) procedures or fertility testing & .79 \\
Fertility drugs & .77 \\
Specific medical diagnoses & .64 \\
Children & .83 \\
Health information or advice in posts & .73 \\
Health information or advice in comments & .72 \\
Outside sources of information & .64 \\
Alternative therapies or alternative medicine & .70 \\
Mistrust of or dissatisfaction with the medical community & .66 \\
Emotional despair, heartbreak, or frustration related to trying to conceive & .75 \\
Emotional despair, heartbreak, or frustration related to trying to adopt & .71 \\
Emotional despair, heartbreak, or frustration related to miscarriage or stillbirth & .71 \\
Emotional despair, heartbreak, or frustration related to other life situations & .67 \\
Sense of community or sisterhood/brotherhood & .64 \\
Emotional gratification from writing or posting & .62 \\
\hline
\end{tabular}




\subsubsection{Blogging Motivations}

Survey participants were asked to rate how important various aspects of blogging were to them using a 4-point rating scale ranging from 1 (not at all important) to 4 (extremely important). Specific item content and descriptive statistics for individual items (including means, medians, modes, standard deviations, minimums, and maximums) can be found in Table 2. Since these descriptive statistics are relevant to the fourth research question, they will be addressed further in the Results section.

Table 2. Summary of item content and descriptive statistics for the importance of aspects of the blogging experience

\begin{tabular}{|c|c|c|c|c|c|c|}
\hline Aspect of the blogging experience & Mean & Median & Mode & $S D$ & Min & $\operatorname{Max}$ \\
\hline Being able to express my thoughts and feelings in writing & 3.71 & 4.00 & 4.00 & .57 & 1.00 & 4.00 \\
\hline Having my thoughts and feelings organized & 3.20 & 3.00 & 3.00 & .81 & 1.00 & 4.00 \\
\hline Having my thoughts and feelings captured in a known location & 3.04 & 3.00 & 4.00 & .93 & 1.00 & 4.00 \\
\hline Knowing others may read what I write & 2.55 & 3.00 & 2.00 & .91 & 1.00 & 4.00 \\
\hline Knowing others may benefit from what I post & 3.26 & 3.00 & 4.00 & .76 & 1.00 & 4.00 \\
\hline Getting comments & 2.21 & 2.00 & 2.00 & .91 & 1.00 & 4.00 \\
\hline Having others link to my blog & 1.79 & 2.00 & 1.00 & .82 & 1.00 & 4.00 \\
\hline Being mentioned by others & 1.69 & 1.00 & 1.00 & .82 & 1.00 & 4.00 \\
\hline Becoming a famous blogger & 1.18 & 1.00 & 1.00 & .46 & 1.00 & 3.00 \\
\hline Making money from blogging & 1.13 & 1.00 & 1.00 & .42 & 1.00 & 3.00 \\
\hline Entertaining my readers & 2.19 & 2.00 & 2.00 & .93 & 1.00 & 4.00 \\
\hline Educating my readers & 2.60 & 3.00 & 3.00 & .93 & 1.00 & 4.00 \\
\hline Touching my readers emotionally & 2.79 & 3.00 & 3.00 & .88 & 1.00 & 4.00 \\
\hline Being funny & 2.12 & 2.00 & 2.00 & .90 & 1.00 & 4.00 \\
\hline Offering health facts or advice to readers & 1.94 & 2.00 & 1.00 & .91 & 1.00 & 4.00 \\
\hline Venting frustrations & 2.92 & 3.00 & 3.00 & .97 & 1.00 & 4.00 \\
\hline Forming an online social support network & 2.81 & 3.00 & 4.00 & 1.11 & 1.00 & 4.00 \\
\hline
\end{tabular}

2.2.3 Self-Reported Blog Content

Survey participants were asked to respond to a series of Yes/No items about the presence of various areas of content on their blogs. Items were highly similar to those addressed in the content analysis (see Table 3 for specific topics assessed and summary statistics). The summary statistics will be described further in the Results section since they partially answer the second research question.

Table 3. Summary of responses to items about blog content

\begin{tabular}{|c|c|c|}
\hline Area of content & $\%$ Yes & $\% \mathrm{No}$ \\
\hline Attempts to get pregnant & 86.73 & 13.27 \\
\hline Attempts to adopt & 30.09 & 69.91 \\
\hline Being pregnant & 73.68 & 26.32 \\
\hline Miscarriage or stillbirth & 56.64 & 43.36 \\
\hline Assisted reproductive technology (ART) procedures or fertility testing & 70.04 & 29.96 \\
\hline Fertility drugs & 70.04 & 29.96 \\
\hline Specific medical diagnoses & 81.50 & 18.50 \\
\hline Parenting or raising children & 78.76 & 21.24 \\
\hline Your own children, stepchildren or foster children & 77.09 & 22.91 \\
\hline Health information or advice & 70.61 & 29.39 \\
\hline Recommending outside sources of information & 72.69 & 27.31 \\
\hline Alternative therapies or alternative medicine & 43.86 & 56.14 \\
\hline Mistrust of or dissatisfaction with the medical community & 50.44 & 49.56 \\
\hline Emotional despair, heartbreak, or frustration related to trying to conceive & 83.33 & 16.67 \\
\hline Emotional despair, heartbreak, or frustration related to trying to adopt & 25.44 & 74.56 \\
\hline Emotional despair, heartbreak, or frustration related to other life situations & 86.34 & 13.66 \\
\hline
\end{tabular}

\subsection{Procedure}

\subsubsection{Content Analysis}

After attending training and achieving adequate agreement with the master coder, coders were assigned to complete written forms regarding blog content within a one-week period (Sunday, January 27, 2013 to Saturday, February 2, 2013). The one-week time period was selected to obtain a sampling of blog content without undergoing the arduous task of coding an entire blog's content (as noted previously, some blogs had been maintained for over five years). Coders first recorded the date of coding, the exact blog title, and the blog URL. Next, they completed three preliminary items. The first preliminary item asked whether the blog was active. For this study, a blog was labeled as active if at least one post appeared within one year of coding. In other words, any blog with at least one post of any length or content type since January 27, 2012, was considered active. Blogs with no posts since that date were designated as dead/dormant/inactive. No further coding occurred for these blogs. 
The second preliminary item addressed whether the blog was fully or partially password-protected or invitation-only. If coders were required to enter login credentials to view the blog or any individual posts, the blog was labeled as password-protected. If the entire blog or posts within the designated coding week were password-protected, no further coding took place. If, however, posts were openly available during the coding week, coders were required to code the content of posts, too.

The third and final preliminary question was included to capture sites that could not be coded for a variety of reasons that included not being an actual blog (i.e., Twitter feeds, commercial websites with no journaling component), not including dates, blogs begun after the designated coding week, broken links, deleted blogs, and blogs seeming to have been hacked or sparking warnings from anti-virus or anti-malware software. Any blog fitting one of these descriptions was not coded further.

Next, for active and openly available dated blogs, coders documented the content of those blogs based on either frequency (continuous count items) of content areas or mention at all (Yes/No items) of content areas. Specific areas of content coded have been listed in the Measures section and can be found in Tables 1 and 3. With the exception of health facts or bits of health advice in comments, coders were trained to examine only the content of posts for all of these Yes/No items.

\subsubsection{Survey}

Bloggers opting to take the survey were allowed to complete the online survey at their convenience. An informed consent page opened the survey, on which participants indicated consent with initials. Participants were informed they could skip any items with which they felt uncomfortable, and all information was kept confidential. The survey and procedures were approved by the university Institutional Review Board (IRB).

\section{Results}

\subsection{Results of the Content Analysis}

Addressing the first research question, out of the original pool of 3527 blogs, 407 (12.20\%) were partially or fully password-protected or invitation only, 1251 (42.59\%) were dead/dormant/inactive (with the most recent post being over a year old), and 199 (5.68\%) were not actually dated blogs (i.e., deleted blogs, hacked sites, undated blogs, or broken links). Out of the 1686 active blogs in the sample (i.e., those with at least one post in the past year), 574 had at least one post during the designated coding week. Four of those blogs, while allowing site access without a password or invitation, had at least one password-protected post during the coding week and therefore could not be coded.

The remaining 570 blogs were coded for the week's content, allowing us to gather information relevant to the second research question. There was a mean of 2.28 posts that week (median $=2$; mode $=1 ; S D=3.08$; range $=1-60)$, with an average of 13.35 comments across all posts (median $=4$; mode $=0 ; S D=43.67$; range $=0-892$ ). Posts for the week included a mean of .74 (median $=0$; mode $=0 ; S D=2.38$; range $=0-22$ ) links to other blog posts. The percentages for Yes/No items are summarized in Table 4. As displayed in Table 4, the most mentioned topic was children with 57.02\% of the included blogs contained some content pertaining to children. The next most common topic was emotional despair, heartbreak or frustration over other life situations, meaning it was not linked to trying to conceive, adoption, or miscarriage or stillbirth (47.72\%). Pregnancy was another popular topic, with $27.19 \%$ mentioning pregnancy and $26.32 \%$ including content about attempts to become pregnant. Refer to Table 4 for response summaries for other types of content, providing us with partial elucidation regarding the second research question. 
Table 4. Results of content analysis of active and openly accessible blogs with content $(N=570)$

\begin{tabular}{lrr}
\hline Area of content & Yes & No \\
\hline Attempts to get pregnant & $150(26.32 \%)$ & $420(73.68 \%)$ \\
Attempts to adopt & $59(10.35 \%)$ & $511(89.65 \%)$ \\
Being pregnant & $155(27.19 \%)$ & $415(72.81 \%)$ \\
Miscarriage or stillbirth & $72(12.63 \%)$ & $498(87.37 \%)$ \\
Assisted reproductive technology (ART) procedures or fertility testing & $125(21.93 \%)$ & $445(78.07 \%)$ \\
Fertility drugs & $77(13.51 \%)$ & $493(86.49 \%)$ \\
Specific medical diagnoses & $76(13.33 \%)$ & $494(86.67 \%)$ \\
Children & $325(57.02 \%)$ & $245(42.98 \%)$ \\
Health information or advice in posts & $133(23.33 \%)$ & $437(76.67 \%)$ \\
Health information or advice in comments & $102(17.89 \%)$ & $468(82.11 \%)$ \\
Recommending outside sources of information & $68(11.93 \%)$ & $502(88.07 \%)$ \\
Alternative therapies or alternative medicine & $34(5.96 \%)$ & $536(94.04 \%)$ \\
Mistrust of or dissatisfaction with the medical community & $45(7.89 \%)$ & $525(92.11 \%)$ \\
Emotional despair, heartbreak, or frustration related to trying to & $124(21.75 \%)$ & $446(78.25 \%)$ \\
conceive & $33(5.79 \%)$ & $537(94.21 \%)$ \\
Emotional despair, heartbreak, or frustration related to trying to adopt & $64(11.23 \%)$ & $506(88.77 \%)$ \\
Emotional despair, heartbreak, or frustration related to miscarriage or & & \\
stillbirth & $272(47.72 \%)$ & $298(52.28 \%)$ \\
Emotional despair, heartbreak, or frustration related to other life & & \\
situations & $126(22.11 \%)$ & $444(77.89 \%)$ \\
Sense of community or sisterhood/brotherhood & $70(12.28 \%)$ & $500(87.72 \%)$ \\
\hline Emotional gratification from writing or posting &
\end{tabular}

3.2 Results for Self-reported Blog Content

To further answer the second research question, Table 3 (mentioned previously in the Measures section) lists the blogger-reported frequencies of specific content based on Yes/No items in the survey. As evident in Table 3, most content areas were reported in at least $50.00 \%$ of blogs, with only "alternative therapies or alternative medicine" (43.86\%), "attempts to adopt" (30.09\%), and "emotional despair, heartbreak, or frustration related to trying to adopt" $(25.44 \%)$ endorsed by less than half of the participants. Most frequently reported content areas were "attempts to get pregnant" (86.73\%), "emotional despair, heartbreak, or frustration related to other life situations" (86.34\%), "emotional despair, heartbreak, or frustration related to trying to conceive" (83.33\%), and "specific medical diagnoses" (81.50\%). For frequencies of other topics, see Table 3. The results presented in Table 3 and 4, taken together, place pregnancy, trying to become pregnant, children, and frustrations as particularly likely topics to appear in FB blogs.

\subsection{Results for Blogging Motivations}

As preliminary clarification of the fourth research question, the descriptive statistics for the importance of particular elements of the blogging experience are summarized in Table 2 (mentioned previously in the Method section). The most highly rated aspect of blogging was "being able to express my thoughts and feelings in writing" $(M=3.71 ; S D=.57)$ with a score approaching 4 or "extremely important." The next most highly rated components were "knowing others may benefit from what I post" $(M=3.26 ; S D=.76)$, "having my thoughts and feelings organized" $(M=3.21 ; S D=.81)$, and "having my thoughts and feelings captured in a known location" $(M=3.04 ; S D=.93)$, all with average scores slightly above 3 or "moderately important." The lowest ranked elements were "becoming a famous blogger" $(M=1.18$; $S D=.46)$ and "making money from blogging" $(M=1.13 ; S D=.42)$, with scores close to 1 or "not at all important." For average rankings of other items, refer to Table 2 .

Given the large numbers of items, before further addressing the fourth research question, data reduction was desirable. A principal components analysis (PCA) with Promax rotation was conducted with the individual items listed in Tables 2 and 5. The Kaiser-Meyer-Olkin (KMO) Measure of Sampling Adequacy was .80, and Bartlett's Test of Sphericity was significant $(p=.00)$. Therefore, data was deemed suitable for PCA. Communalities indicated all items exhibited extraction values of at least .50. Factor loadings from the pattern matrix are summarized in Table 5. Using an eigenvalue cutoff of 1.00 and pattern matrix component loading cutoff of .50, results supported the presence of up to six components. Given the PCA results and past research (Huang et al., 2007; Nardi et al., 2004b), seven variables for blogging motivations were created, with six variables created from multiple items and one reflecting a single item score. Composite variables were computed with the mean rather than sum so as to retain the scaling of 1 (not at all important) to 4 (extremely important). Based on the PCA results (see Table 5), four composite variables (gaining attention, benefitting others, becoming a career blogger, and entertaining others) were created using the mean of items with a factor loading of .50 or higher. The variable "gaining attention" comprised the mean of "knowing others may read what I write," "getting comments," "having others link to my blog," and "being mentioned by others." The variable "benefitting others" was computed from "knowing others may benefit from what I post," "educating my readers," 
"touching my readers emotionally," and "offering health facts or advice to readers." The "becoming a career blogger" variable was created by averaging "becoming a famous blogger" and "making money from blogging." The next variable, "entertaining others" was made up of the mean of "entertaining my readers" and "being funny."

A fifth composite variable, "documenting life events," was calculated using the mean of "having my thoughts and feelings organized" and "having my thought and feelings captured in a known location." While the item "being able to express my thoughts and feelings in writing" loaded on the same factor with a high enough value for inclusion (.58), this item exhibited factor loadings over .50 for both the fifth and sixth factors. The loadings for the sixth factor, as a whole, fit less well with past research than the first five factors. Based on the work of Huang and colleagues (2007) we created a sixth variable labeled "self-expression" calculated from the mean of "venting frustrations" and "being able to express my thoughts and feelings in writing." The variable "forming an online social support network" was left as a single-item variable. Therefore, seven blogging motivation variables were included in subsequent analyses: gaining attention, benefitting others, becoming a career blogger, entertaining others, documenting life events, self-expression and forming an online social support network.

Table 5. Summary of factor loadings from principal components analysis of blogging motivation items (Promax rotation used)

\begin{tabular}{|c|c|c|c|c|c|c|}
\hline Aspect of the blogging experience & GA & $\mathrm{BO}$ & $\mathrm{CB}$ & EO & DLE & $\mathrm{SE}$ \\
\hline Being able to express my thoughts and feelings in writing & -.18 & .07 & .10 & .06 & .58 & .53 \\
\hline Having my thoughts and feelings organized & .14 & .04 & .03 & -.18 & .77 & -.14 \\
\hline $\begin{array}{l}\text { Having my thoughts and feelings captured in a known } \\
\text { location }\end{array}$ & .10 & -.08 & -.20 & .14 & .76 & -.01 \\
\hline Knowing others may read what I write & .67 & .07 & -.15 & .11 & .03 & .13 \\
\hline Knowing others may benefit from what I post & .25 & .70 & -.12 & -.12 & -.11 & .15 \\
\hline Getting comments & .91 & -.26 & -.11 & .19 & -.04 & .14 \\
\hline Having others link to my blog & .76 & .08 & .11 & -.09 & .15 & -.09 \\
\hline Being mentioned by others & .71 & .03 & .27 & -.09 & .11 & -.15 \\
\hline Becoming a famous blogger & .10 & .00 & .78 & .11 & -.06 & .00 \\
\hline Making money from blogging & -.09 & -.04 & .94 & .01 & -.08 & .13 \\
\hline Entertaining my readers & .16 & .02 & .06 & .83 & -.07 & -.07 \\
\hline Educating my readers & -.14 & .93 & -.03 & .12 & -.02 & -.08 \\
\hline Touching my readers emotionally & .14 & .60 & .06 & .15 & .01 & -.04 \\
\hline Being funny & -.01 & .12 & .05 & .83 & .06 & -.02 \\
\hline Offering health facts or advice to readers & -.16 & .83 & .03 & .00 & .05 & -.04 \\
\hline Venting frustrations & .00 & -.13 & .09 & -.03 & -.01 & .87 \\
\hline Forming an online social support network & .33 & .19 & -.01 & -.13 & -.16 & .60 \\
\hline
\end{tabular}

Description. $\mathrm{GA}=$ Gaining Attention, $\mathrm{BO}=$ Benefitting Others, $\mathrm{CB}=$ Becoming a Career Blogger, $\mathrm{EO}=$ Entertaining Others, DLE $=$ Documenting Life Events, $\mathrm{SE}=$ Self-expression; A bolded value indicates an item was included in a composite variable in later analyses. One item, forming an online social support network, was retained as a single-item measure. All variables created, whether composed of multiple items or a single item, retained the original scaling of 1 (not at all important) to 4 (extremely important).

To further address the fourth research question, the means and standard deviations of the final seven blogging motivation variables are listed in Table 6 with the bivariate correlations among them. As evident in Table 6 , self-expression had the highest mean rating $(M=3.32 ; S D=.63)$, followed by documenting life events $(M=3.12 ; S D$ $=.72)$. The lowest rated motivation was becoming a career blogger $(M=1.16 ; S D=.39)$. For other means, refer to Table 6. As apparent in Table 6, many of the final blogging motivation variables were significantly correlated.

Table 6. Descriptive statistics and correlations among final blogging motivation variables

\begin{tabular}{|c|c|c|c|c|c|c|c|}
\hline Item & Mean $(S D)$ & GA & $\mathrm{BO}$ & CB & EO & DLE & SE \\
\hline Gaining attention & $2.06(.70)$ & - & & & & & \\
\hline Benefitting others & $2.65(.68)$ & $.50 * *$ & - & & & & \\
\hline Becoming a career blogger & $1.16(.39)$ & $.36 * *$ & $.41 * *$ & - & & & \\
\hline Entertaining others & $2.16(.84)$ & $.41 * *$ & $.50 * *$ & $.39 * *$ & - & & \\
\hline Documenting life events & $3.12(.72)$ & $.28 * *$ & $.16^{*}$ & .07 & .10 & - & \\
\hline Self-expression & $3.32(.72)$ & $.19 * *$ & $.14 *$ & .02 & .06 & $.17 *$ & - \\
\hline Forming an online social support network & $2.81(1.11)$ & $.36 * *$ & $.32 * *$ & .12 & .13 & .06 & $.38 * *$ \\
\hline
\end{tabular}

Description. $\mathrm{GA}=$ Gaining Attention, $\mathrm{BO}=$ Benefitting Others, $\mathrm{CB}=$ Becoming a Career Blogger, $\mathrm{EO}=$ Entertaining Others, $\mathrm{DLE}=$ Documenting Life Events, $\mathrm{SE}=$ Self-expression $; * p<.05 ; * * p<.01$

To address the fifth research question by exploring differences in blogging motivations among the three largest self-reported blogger subgroup categories (those having experienced infertility, $n=169$; those having experienced pregnancy loss, $n=104$; and those who were already parents, $n=151$ ), a multivariate analysis of variance (MANOVA) 
was conducted with all seven blogging motivation variables included as dependent variables. MANOVA was conducted rather than a series of factorial ANOVA due to significant correlation between most of the six dependent variables $(r$ $=.01$ to $r=.50 ; p<.05$ for 14 out of 21 correlations; see Table 6 ). The assumption of homogeneity of covariances was examined with Box's test of equality of covariance matrices, and results were significant $(p=.02)$, suggesting the assumption had been violated. Box's test is known to be highly sensitive and unequal cell sizes can increases the likelihood of getting significant results. Furthermore, the assumption of normality did appear to be violated for all dependent variables based on significant Kolmogorov-Smirnov and Shapiro-Wilk tests. Since ANOVA procedures are considered robust to violations of these assumptions, analyses proceeded but results should be interpreted with caution. Only one main effect was statistically significant. Specifically, bloggers having experienced infertility differed significantly $(p<.01)$ from those who not reporting experience with infertility (Wilks' $\Lambda=.92 ; F=3.41 ; p=.00$; partial $\eta^{2}=.08$ or medium effect size). For pregnancy loss (Wilks' $\Lambda=.98 ; F=.88 ; p=.51$ ) and parenting (Wilks' $\Lambda$ $=.96 ; F=1.75 ; p=.11)$, results were not significant, and no interaction effects were significant.

Follow-up tests were conducted using a series of univariate ANOVA. There were more significant effects supported in the ANOVA than the MANOVA, but these effects will not be interpreted or discussed since doing so when the MANOVA result was not significant is indicative of Type I error. For the infertility main effect, differences were significant $(p<.05)$ for educating others $\left(F=1.96 ; p=.04\right.$; partial $\eta^{2}=.02$ or small effect size $)$ and self-expression $(F$ $=4.15 ; p=.001 ;$ partial $\eta^{2}=.05$ or small effect size). For both blogging motivations, mean ratings were higher for participants having experienced infertility.

\section{Discussion}

\subsection{How Accessible and Active Are FB Blogs?}

Based on our content analysis, an internet user seeking information or personal stories about FB would likely encounter many dead/dormant/inactive or password-protected sites before finding an actual active blog. There has been prior evidence of tremendous diversity in blogging frequency among bloggers (see Nardi et al., 2004b), indicating even active blogs may not often contain new posts. Indeed, out of the 3527 blogs originally identified, only 1686 were active, and only 570 had new and openly accessible posts within the designated coding week. Blogs with more frequent posts would provide more opportunities to disseminate information, and bloggers posting more often about health conditions report greater levels of social support (Rains \& Keating, 2011). Still, it is not difficult to explain why many FB blogs would be inactive or password-protected. Those successfully conceiving or adopting a child (or children) may become too busy with parenting duties to maintain their blog. Alternatively, they may feel their story is complete, especially if the blog was set up as a "trying to conceive" blog. Another explanation is that some may worry disclosure has shifted to overdisclosure or fear retribution from IRL individuals mentioned in their blog, prompting a halt in posting or introducing password-protection.

\subsection{What Sort of Content Would a Reader Encounter upon Finding an Open and Active FB Blog?}

Taken together, the results of the content analysis and the survey provide some clarification about the kind of information readers would come across when finding one of these blogs. The frequency of content documented in the content analysis differed from what was reported in the survey of active bloggers in that topics generally appeared to occur more frequently based on survey data. This can be explained by how data were collected. While content coders were instructed to examine content during a single week, bloggers participating in the survey were able to look back upon their entire posting history. In addition, bloggers themselves would have had access to both the words typed and their intended meaning, whereas content coders could only make decisions based on the written words and whether they matched with descriptions in the coding manual. Importantly, bloggers opting to take the survey may represent a unique group. They would need to have been checking their blog comments or e-mail account often enough to know about the survey, and be motivated enough to open the survey at all. Less active or more reticent or private bloggers may have been disproportionately excluded. These factors may have led to the higher frequency of content areas based on self-report versus time-limited objective content analysis. Nonetheless, both means of data collection indicated FB blogs discuss a variety of content.

The blogs were particularly likely to include posts about trying to conceive, pregnancy, and raising children already conceived or adopted. In fact, the finding that so many blogs discussed rearing children may suggest a particular source of appeal for FB blogs. That is, many transition from trying to become parents to actually being parents. Readers just beginning their quest to become parents and those struggling to maintain hope in the face of infertility, pregnancy loss, or unsuccessful adoption attempts may garner optimism from perusing archived posts to read how a particular blogger progressed from "trying" to "parenting." Reading that the blogger currently posting cheery holiday pictures of her toddler previously experienced two miscarriages and five unsuccessful ART procedures may provide hope and patience that a fact-focused visit with a medical professional would not as likely produce. 
Blogs may provide the additional benefit of allowing readers to select bloggers having experience with less frequently discussed possibilities in the journey to parenthood. In both the content analysis and the survey, miscarriage/stillbirth and adoption emerged as less common topics than trying to become pregnant or being pregnant. Readers seeking information or support related to these less common situations may feel isolated when visiting sites (blogs or other resources) discussing FB with a focus on conception of a viable pregnancy. The blogging community, however allows readers to pick and choose, through blog aggregators, categorized blogrolls, or simply bookmarking which blogs are the best fit for them to follow.

Similarly, bloggers undergoing ART, struggling with a specific medical diagnosis, and/or taking fertility drugs may have few IRL friends to discuss their unique experiences with. Blogging, in this case, may provide the opportunity to document the minutia of procedures and testing in a forum for those who are likely to understand the vocabulary and nuances that accompany procedures and testing. While mention of fertility drugs and specific medical diagnoses were comparably lower in the content analysis than the survey, this may be due to the timeline of coding. A blogger may not mention or label their diagnosis every time they post, but instead focus on new testing, procedures, or symptoms. In regards to fertility drugs, a given week may not be a drug-taking week since one does not typically take them continuously. Alternatively, a blogger may have previously taken fertility drugs but had stopped by the time of the coding week.

While they were indicated the lowest frequency topics in both the content analysis and survey, general dissatisfaction with the medical community and alternative treatment options were both mentioned in at least some blogs. For some FB bloggers, their sites may provide a means of expressing how the medical community has failed to meet their needs. Additionally, they may be able to document their exploration of alternative therapies and learn of unexplored options through reader comments. Offering health facts or advice in both posts and comments and recommending outside sources of information emerged as moderately popular topics, and no distinction was made as to whether the health facts or advice or outside sources were evidence-based or alternative in nature. The fact that these topics are discussed at all in a group heavily populated by those currently or trying to become pregnant is cause for concern. Blogs are not required to provide academic credentials or cite empirical research supporting the efficacy or safety of any remedy or therapy presented in either posts or comments. Bloggers and their readers may be unaware of the dangers of certain alternative treatments during pregnancy (e.g., Ernst, 2002). In this case, the accessibility of blogs may more of a disadvantage than an advantage. While readers may think twice about trying untested alternative therapies after reading about a blogger's lack of success or harmful side effects with one, it is possible that some readers would consider trying it themselves simply from learning of its existence.

The content analysis and survey also revealed both positive and negative emotional aspects of blogging. Many blogs documented the bloggers' frustrations with either trying to become a parent or with other elements of life. In contrast, discussing these negative experiences may be beneficial, as the content analysis indicated some bloggers experienced either emotional catharsis from writing or a sense of community from feedback to posts. All in all, both the content analysis and survey painted a set of both homogenous and heterogeneous blogs, all united by the theme of building or maintaining families while distinguished by specific experiences in working toward distinct goals.

\subsection{Who Tends to Keep a FB Blog?}

After gaining knowledge about the blogs, we sought to learn more about the bloggers. Our results were consistent with previous non-empirically-based descriptions describing a close-knit web of predominately White females keeping personal journal-style FB blogs (Miller, 2008). In our sample of bloggers completing the survey, diversity was notably lacking, with the overwhelming majority of respondents being female and Caucasian. While some reported contributing to group-hosted blogs, most kept an online personal journal. There was more diversity in terms of age, with participants as young as 19 and as old as 59, with the average age falling in the thirties. While most bloggers had experience with at least one of the barriers to FB of infertility or pregnancy loss, there were respondents representing a wide variety of participants in the FB process, including same-sex couples, single adults, and adoptive parents.

\subsection{What Motivates an Individual to Blog about Family Building?}

The survey provided further insight into individual motivations for blogging. First and foremost, the participating FB bloggers seemed to blog to satisfy needs for self-expression and documenting their life experiences. That is, bloggers reported valuing the opportunity to write about their experiences and feelings and having one convenient forum for such expression. Nardi and colleagues (2004b) have articulated that blogs are forums well-suited for self-expression and keeping a log of life's happenings since only those interested in what is written will read, and the reverse chronological order allows readers to do so at a time convenient to them. Likewise, the diary format additionally may simply call for processing thoughts, feelings, and reactions to life events.

Consistent with previous research (e.g., Miller \& Pole, 2010), the next most prominent motivation was building an 
online network of social support. It remains to be seen whether FB bloggers are satisfied with the support networks built out of blogging, and how these online networks compare to networks IRL. Female bloggers have previously reported less intimacy within online friendships compared to those IRL (Bane, Cornish, Erspamer, \& Kampman, 2010), but perceived stigma for certain FB topics might alter trust and openness for friendships IRL. In addition, there is evidence that new mothers experience improved connection with the outside world and increased social support when they blog about pregnancy and parenthood (McDaniel, Coyne, \& Holmes, 2012). FB blogging may, therefore, allow for establishing social support difficult to obtain IRL for individuals feeling isolated or misunderstood.

While rated as less important, bloggers also reported striving to provide helpful insight, information, and entertainment, as if providing a service. Miller and Pole (2010) similarly reported health bloggers aiming to provide useful information to readers, but blogging motivation took a back seat to forming online support networks. Attracting attention was endorsed as less important, and despite certain media portrayals of the "mommy blogger" as money-making celebrity (see Belkin, 2011; Murphy, 2011), these blogging motivations emerged as least important. In other words, the two lowest rated motivations were highly self-oriented in nature, lacking the "we are in this together" notion implicit in more highly rated motivations. As a group, participants reported blogging to connect with others to make sense of their own situations while potentially helping others find useful information or amusement while reading about their attempts to do so.

\subsection{Do Blogging Motivations Differ among Subgroups of FB Bloggers?}

Given the apparent presence of subgroups of FB bloggers (i.e., those having experienced infertility, those having experienced pregnancy loss, and bloggers already parenting), the investigators sought to explore potential differences in blogging motivations between these subgroups. For most comparisons, differences were not supported, perhaps because FB bloggers are fairly homogeneous in their motivations for keeping up a blog about their feelings and experiences, regardless of which heading they fall under within a blogroll. The one exception was that bloggers having experienced infertility endorsed educating others and self-expression more highly than bloggers who had not experienced infertility. Bloggers with personal experience with the frustrations and isolation of infertility may be particularly drawn to blogging as a means of passing on the knowledge collected over the course of their struggles and to articulate feelings and insights brought on by those struggles. Further investigation, is, of course, needed to more fully establish and explain such a difference in blogging motivations in those FB bloggers having experienced infertility.

\subsection{Limitations and Directions for Future Research}

The current study should be viewed as a starting point in research into FB blogging. The results are preliminary and by no means conclusive, partly because the investigation possessed several noteworthy limitations. First, while attempts were made to be highly exhaustive in including blogs, only blogs locatable through Google, blogrolls, or blog listing websites were included. Some blogging services allow bloggers to block their site from Google searching, therefore, some blogs may have been left out. In addition, we only coded one week's worth of content in the content analysis. The time frame was, admittedly, rather arbitrary. Some blogs may have appeared minimally active because we inadvertently selected a "slow" blogging week. Furthermore, only dated blogs written in English were included in the sample. In addition, as noted previously, the participants in the survey were likely not representative of all family building bloggers. They probably possessed a high level of motivation and investment in blogging. Finally, the results regarding group differences involved unequal cell sizes and skewed data, meaning results should be interpreted cautiously and with skepticism without further replication.

Future research is clearly called for, and it should work to include more blogs, if possible, and the timeline for content coding may best be expanded. Furthermore, if replicating the survey portion of the study, efforts to increase participation should be undertaken. Much of this study was descriptive in nature, especially in regard to blog content, and further hypothesis testing would greatly increase understanding of the family building blogosphere. One question not addressed in this study would be to examine whether personal characteristics of bloggers (i.e., education level, religion, income) not collected in the current study are associated with different rates of posting or content or different blogging motivations. Comparisons were not made between different genders or races because of the lack of diversity in the sample, but it would certainly be interesting to learn more about why men and non-White individuals seem unlikely to blog. Alternatively, researchers could track content over an extended period of time to document change in content or frequency of posts after specific events (i.e., childbirth, loss, unsuccessful procedure or adoption) occur. A final recommendation would be to undergo in-depth interviews with family building bloggers (similar to Nardi et al., 2004b) to more fully explore blogging motivations and benefits of blogging.

\subsection{Summary and Conclusions}

Results of this study, while preliminary, indicate that FB blogs tend to be maintained by White females, possibly limiting their appeal to men and non-White individuals with questions or concerns about the FB process. These blogs 
appear to often become inactive or password-protected, thus limiting potential readers' ability to access current content. When presenting new posts, FB blogs tend to discuss a variety of topics, with particular emphasis on topics related to children, pregnancy, or trying to become pregnant. Bloggers seem to particularly value documenting the events and their reactions to their FB experiences, benefitting readers with what they post, and the social support network that builds out of maintaining a blog. Experience with infertility may affect blogging motivations such that education and self-expression are particularly important.

FB blogs are an easily accessible source of information and support for those striving to expand or maintain a family. Some caution is recommended, however, since information presented is not required to be fact-checked or evidence-based. With this caveat in mind, medical professionals, mental health professionals, adoption professionals, and potential parents would benefit from greater awareness of FB blogs and their potential to ease the sometimes long and arduous process of building or maintaining a family. FB blogs may provide a supplement to information provided by professionals and a means of connecting with others or making sense of one's own struggles in a safe space allowing for anonymity and the blogger or reader's control over topics covered.

\section{References}

Alati-it, J., \& Pinches, A. (2014). Consumer health information resources on pregnancy, birth, and fertility. Journal of the Canadian Health Libraries Association, 34, 112-114. http://dx.doi.org/10.5596/c13-031

Bane, C. M., Cornish, M., Erspamer, N., \& Kampman, L. (2010). Self-disclosure through weblogs and perceptions of online and "real-life" friendships among female bloggers. Cyberpsychology, Behavior, and Social Networking, 13, 131-139. http://dx.doi.org/10.1089/cyber.2009.0174

Belkin, L. (2011). Queen of the mommy bloggers. The New York Times, 26-31.

Cousineau, T. M., Lord, S. E., Seibring, A. R., Corsini, E. A., Viders, J. C., \& Lakhani, S. R. (2004). A multimedia psychosocial support program for couples receiving infertility treatment: a feasibility study. Fertility and Sterility, 81, 532-538. http://dx.doi.org/10.1016/j.fertnstert.2003.08.021

Ernst, E. (2002). Herbal medicinal products during pregnancy: are they safe? BJOG: An International Journal of Obstetrics \& Gynaecology, 109, 227-235. http://dx.doi.org/10.1111/j.1471-0528.2002.t01-1-01009.x

Ford, M. (2015). A whole lot of blogging - brought to you sorted and filed. http://www.stirrup-queens.com/a-whole-lot-of-blogging-brought-to-you-sorted-and-filed/

Gold, K. J., Boggs, M. E., Mugisha, E., \& Palladino, C. L. (2012). Internet message boards for pregnancy loss: Who's on-line and why? Women's Health Issues, 22, e67-e72. http://dx.doi.org/10.1016/j.whi.2011.07.006

Himmel, W., Meyer, J., Kochen, M. M., \& Michelmann, H. W. (2005). Information needs and visitors' experience of an internet expert forum on infertility. Journal of Medical Internet Research, 7, e20. http://dx.doi.org/10.2196/jmir.7.2.e20

Huang, C. Y., Shen, Y. Z., Lin, H. X., \& Chang, S. S. (2007). Bloggers' motivations and behaviors: a model. Journal of Advertising Research, 47, 472-484. http://dx.doi.org/10.2501/S0021849907070493

Knoll, M., \& Bronstein, J. (2014). An examination of the information disclosure behavior of infertility bloggers: Patterns of self-disclosure and anonymity. Aslib Journal of Information Management, 66, 175-201. http://dx.doi.org/10.1108/AJIM-06-2013-0055

Malik, S. H., \& Coulson, N. (2008). The male experience of infertility: A thematic analysis of an online infertility support group bulletin board. Journal of Reproductive and Infant Psychology, 26, 18-30. http://dx.doi.org/10.1080/02646830701759777

McDaniel, B. T., Coyne, S. M., \& Holmes, E. K. (2012). New mothers and media use: Associations between blogging, social networking, and maternal well-being. Maternal and Child Health Journal, 16, 1509-1517. http://dx.doi.org/10.1007/s10995-011-0918-2

McLellan, F. (1998). "Like hunger, like thirst": patients, journals, and the internet. Lancet, 352, Suppl 2SII39-SII43. http://dx.doi.org/10.1016/S0140-6736(98)90301-4

Miller, C. (2008). Blogging infertility. New Atlantis, 19, 79-90.

Miller, E. A., \& Pole, A. (2010). Diagnosis blog: checking up on health blogs in the blogosphere. American Journal of Public Health, 100, 1514-1519. http://dx.doi.org/10.2105/AJPH.2009.175125

Murphy, K. (2011). My blog is also paying my bills. The New York Times, B10

Nardi, B. A., Schiano, D. J., \& Gumbrecht, M. (2004a). Blogging as social activity, or, would you let 900 million people 
read your diary? In Proceedings of the 2004 ACM conference on Computer Supported Cooperative Work (222-231). Chicago, IL: ACM.

Nardi, B. A., Schiano, D. J., Gumbrecht, M., \& Swartz, L. (2004b). Why we blog. Communications of the ACM, 47, 41-46.

Neal, D. M., \& McKenzie, P. J. (2011). Putting the pieces together: endometriosis blogs, cognitive authority, and collaborative information behavior. Journal of the Medical Library Association, 99, 127-134. http://dx.doi.org/10.3163/1536-5050.99.2.004

Okamura, K., Bernstein, J., \& Fidler, A. T. (2002). Assessing the quality of infertility resources on the World Wide Web: tools to guide clients through the maze of fact and fiction. Journal of Midwifery \& Women's Health, 47, 264-268. http://dx.doi.org/10.1016/S1526-9523(02)00260-X

Rains, S. A., \& Keating, D. M. (2011). The social dimension of blogging about health: Health blogging, social support, and well-being. Communication Monographs, 78, 511-534. http://dx.doi.org/10.1080/03637751.2011.618142

Ratliff, C. (2009). Policing miscarriage: infertility blogging, rhetorical enclaves, and the case of house bill 1677. WSQ: Women's Studies Quarterly, 37, 125-145.

Ressler, P. K., Bradshaw, Y. S., Gualtieri, L., \& Chui, K. K. H. (2012). Communicating the experience of chronic pain and illness through blogging. Journal of Medical Internet Research, 14, e143. http://dx.doi.org/10.2196/jmir.2002

Rousson, V., Gasser, T., \& Seifert, B. (2002). Assessing intrarater, interrater and test-retest reliability of continuous measurements. Statistics in Medicine, 21, 3431-3446. http://dx.doi.org/10.1002/sim.1253

Schiano, D. J., Nardi, B. A., Gumbrecht, M., \& Swartz, L. (2004, April). Blogging by the rest of us. In CHI 2004 Extended Abstracts on Human Factors in Computing Systems (pp. 1143-1146). ACM.

Slade, P., O'Neill, C., Simpson, A. J., \& Lashen, H. (2007). The relationship between perceived stigma, disclosure patterns, support and distress in new attendees at an infertility clinic. Human Reproduction, 22, 2309-2317. http://dx.doi.org/10.1093/humrep/dem115

Striff, E. (2005). "Infertile me:" The public performance of fertility treatments in internet weblogs. Women \& Performance: A Journal of Feminist Theory, 15, 189-206. http://dx.doi.org/10.1080/07407700508571511

Viera, A. J., \& Garrett, J. M. (2005). Understanding interobserver agreement: the kappa statistic. Family Medicine, 37, 360-363.

Weissman, A., Gotlieb, L., Ward, S., Greenblatt, E., \& Casper, R. F. (2000). Use of the Internet by infertile couples. Fertility and Sterility, 73, 1179-1182. http://dx.doi.org/10.1016/S0015-0282(00)00515-X

Welbourne, J. L., Blanchard, A. L., \& Boughton, M. D. (2009, June). Supportive communication, sense of virtual community and health outcomes in online infertility groups. In Proceedings of the Fourth International Conference on Communities and Technologies (pp. 31-40). ACM.

Wischmann, T. (2008). Implications of psychosocial support in infertility--A critical appraisal. Journal of Psychosomatic Obstetrics \& Gynecology, 29, 83-90. http://dx.doi.org/10.1080/01674820701817870

Ziebland, S., \& Wyke, S. (2012). Health and illness in a connected world: How might sharing experiences on the internet affect people's health? The Millbank Quarterly, 90, 219-249.

http://dx.doi.org/10.1111/j.1468-0009.2012.00662.x

\section{(c) $\mathrm{EY}$}

This work is licensed under a Creative Commons Attribution 3.0 License. 